\title{
¿Se infectan más los catéteres tunelizados para hemodiálisis cuando los pacientes ingresan en el hospital?
}

\author{
Isidro Sánchez Villar, Víctor Lorenzo Sellares \\ Hospital Universitario de Canarias. España
}

Como citar este artículo: Sánchez-Villar I, Lorenzo-Sellares V. ¿Se infectan más los catéteres tunelizados para diálisis cuando los pacientes ingresan en el hospital?. Enferm Nefrol. 2019 Jul-Sep;22(3):266-73

\section{Resumen}

La infección de los catéteres para hemodiálisis constituye una de las causas de mayor morbimortalidad. La hipótesis intuitiva de que los catéteres se infectan más durante la hospitalización no ha sido verificada en la literatura.

Objetivo: Conocer si la hospitalización es un factor de riesgo de bacteriemia relacionada con catéter.

Material y Método: Análisis retrospectivo de las bacteriemias asociadas a catéter durante un período de 4 años de un centro periférico extrahospitalario de hemodiálisis dependiente de un Servicio de Nefrología de un hospital de tercer nivel durante el período 2014-2017. Se recogieron los tiempos de empleo de catéteres y las bacteriemias relacionadas con catéter divididos en 2 escenarios: Hospital y centro periférico.

Se aplicó el Modelo de Regresión de Poisson para comparar las bacteriemias Hospital vs centro periférico. Un mismo paciente pudo padecer bacteriemia más de una vez y en los dos escenarios.

Resultados: Se emplearon 361 catéteres en 174 pacientes con tiempo total de uso 91.491 días, con 103 episodios de bacteriemia.

Correspondencia:

Isidro Sánchez Villar

Servicio de Nefrología. Hospital Universitario de Canarias C/ Ofra s/n. 38320 La Laguna. Santa Cruz deTenerife E-mail: isvillar@gmail.com
Tasas de bacteriemias: hospitalizado 7,81/1000 días/ catéter y centro periférico 0,81/1000 días/catéter.

El riesgo de sufrir bacteriemia se multiplicó por 9,6 durante la hospitalización vs centro periférico $(p<0,001)$.

Conclusiones: El riesgo de bacteriemia relacionada con catéter aumenta cuando el paciente está hospitalizado. Las circunstancias relacionadas con la hospitalización son factores de riesgo. Aunque la mayor comorbilidad de estos pacientes puede explicar en parte la mayor incidencia de bacteriemias, la optimización de los cuidados de enfermería -para personal no habituado- es el espacio de mejora del que nos advierten los resultados.

PALABRAS CLAVE: hemodiálisis; catéter venoso central; bacteriemia; hospitalización.

\section{Are tunneled catheters for hemodialysis more infected when patients are admitted to the hospital?}

\section{Abstract}

Infection of catheters for hemodialysis is one of the major causes of morbidity and mortality. The intuitive hypothesis that catheters become more infected during hospitalization has not been verified in the literature.

Objective: To know if hospitalization is a risk factor for catheter-related bacteraemia. 
Material and Method: Retrospective analysis of catheter-associated bacteraemias in dialysis center dependent on a Nephrology Department of a third level hospital during the 2014-2017 period.

Catheter use times and catheter-related bacteraemias were divided into 2 scenarios: hospital and dialysis center.

The Poisson Regression Model was applied to compare bacteremia in hospital and dialysis center. The same patient could suffer bacteremia more than once and in both scenarios.

Results: 361 catheters were used in 174 patients with a total time of use of 91,491 days, with 103 episodes of bacteraemia.

Bacteremia rates: in hospital was 7.81/1000 days/ catheter and in dialysis center was $0.81 / 1000$ days/ catheter.

The risk of bacteraemia was multiplied by 9.6 during hospitalization versus dialysis center $(p<0.001)$.

Conclusions: The risk of catheter-related bacteraemia increases when patients are hospitalized. Circumstances related to hospitalization are risk factors. Although the greater comorbidity of these patients may partly explain the higher incidence of bacteraemias, the optimization of nursing care -for uninhabited personnel- is the focus of improvement, according to our results.

KEYWORDS: hemodialysis; central venous catheter; bacteraemia; hospitalization.

\section{Introducción}

Los profesionales que nos dedicamos al cuidado del paciente renal en tratamiento con hemodiálisis (HD) sabemos que la utilización del catéter venoso central (CVC) es la alternativa a la fístula arteriovenosa (FAV). La literatura científica y las guías clínicas de accesos vasculares para HD indican que el uso de la FAV debe ser la primera opción ${ }^{1-5}$. A pesar de ello, los registros muestran que en nuestro entorno el uso de CVC continúa en ascenso6-8. Sabemos que la BRC es una de las complicaciones que con mayor frecuencia limita la vida útil de un CVC y persiste como un problema mayor, siendo una de las causas de mayor morbimortalidad y hospitalización en los pacientes en tratamiento renal sustitutivo. Con esta realidad, hay una especial sensibilidad para el manejo del CVC y prevención de las BRC. Esto se traduce en el uso de procedimientos de monitorización y vigilancia de los accesos vasculares en las unidades de HD H,9-17 $^{3}$ La observación intuitiva nos dice que los CVC se infectan más (bacteriemias documentadas) cuando el paciente está hospitalizado que cuando se realiza la HD en su CP. No obstante, no hemos encontrado literatura que avale directamente esta afirmación. Puesto que en nuestro medio, aproximadamente el $50 \%$ de los pacientes en programa de HD ingresa al menos una vez al año, y de ellos el 51\% son portadores de $\mathrm{CVC}^{17}$ nuestro objetivo principal fue conocer si la hospitalización de los pacientes portadores de CVC es un factor de riesgo de bacteriemia.

\section{Pacientes y Método}

Análisis retrospectivo de las bacteriemias asociadas a CVC durante un período de 4 años (2014-2017) en pacientes de un centro de HD periférico dependiente de un hospital de tercer nivel. Se compararon las que sucedieron en $\mathrm{CP}$ frente a las que aparecieron durante la hospitalización.

Criterios de inclusión: Pacientes portadores de CVC tunelizado yugular, que se dializaban en el CP de HD del Hospital Universitario de Canarias.

Variables de estudio: Edad, sexo, enfermedad de base (DM si/no), tiempo en HD, $n^{0}$ de CVC/paciente, $n^{\circ}$ y tiempo de hospitalizaciones, tiempo en HD, tiempo de CVC, sellado del CVC, $\mathrm{n}^{\circ}$ de hemocultivos y resultados.

\section{Variable de resultado: BRC}

Los datos se recogieron de la historia clínica electrónica de cada paciente y se volcaron en hoja de Excel.

Protocolo de cura del CVC: El antiséptico utilizado fue solución de clorhexidina alcohólica $2 \%$. El apósito usado fue el IV3000 9x12 cm de formato transparente, de poliuretano, permeable al vapor de agua, y resistente al agua e impermeable a los líquidos, bacterias y virus. El procedimiento fue de cura plana y se realizó con material estéril y mascarilla. La cura fue semanal, a excepción de que el apósito no estuviera íntegro o hubiera un orificio de inserción del CVC dudoso. Se empleó el mismo en hospital y CP). 
Protocolo de sellado de CVC: Se realizó tras cualquier uso del CVC. El procedimiento se realizó con técnica aséptica, material estéril y mascarilla. Antes de realizar el sellado se limpiaron las ramas con alcohol al $70 \%$. Se realizó lavado con $10 \mathrm{ml}$ de suero salino $0,9 \%$ en cada luz en embolada rápida para arrastrar cualquier resto de sangre. Posteriormente, se perfundió el anticoagulante de forma lenta para que el sellado se mantuviese en el CVC. La cantidad de anticoagulante fue la indicada en cada una de las ramas del propio CVC. Se finalizaba con la colocación del tapón estéril.

El sellado en los pacientes hospitalizados se realizó con heparina $5 \%$, y fue el mismo durante todo el período de estudio.

El sellado en el CP se efectuó también con heparina 5\% durante el período 2014 y 2015; y con Citra-Lock ${ }^{\mathrm{TM}}$ (citrato trisódico 4\%) en el período 2016 y 2017.

Definición de BRC: Aparición de fiebre y crecimiento en hemocultivo, sin evidencia clínica de otro foco de infección. La tasa de BRC se calculó como número de BRCX1000 días de CVC.

\section{Tratamiento estadístico:}

Las variables categóricas se han descrito con frecuencias y porcentajes, las variables contínuas con medias \pm desviación estándar, valores máximos y mínimos, y mediana e intervalo de confianza (IC), si la distribución de los valores no era uniforme. Se utilizó la t-Student para relacionar la BRC con las variables cuantitativas y el Chi-cuadrado para relacionar las variables cualitativas, para ello se utilizó el paquete estadístico SPSS 19. Un valor de $p<0,05$ fue considerado estadísticamente significativo.

Se aplicó el Modelo de Regresión de Poisson, del programa estadístico Stata, para comparar las bacteriemias en CP vs en hospital, ajustadas a tiempo en riesgo. Un mismo paciente pudo padecer bacteriemia más de una vez y en los dos escenarios.

\section{Resultados}

En la Tabla 1 se presentan las características de la población estudiada, total y segmentada, en función de la aparición o no de BRC; y en el escenario donde se produjo, Hospital o CP.

Tabla 1. Características de la población de estudio total y segmentada en función de la aparición o no de BRC y en el escenario donde se produjo.

\begin{tabular}{|c|c|c|c|c|c|}
\hline & & \multicolumn{2}{|c|}{ BACTERIEMIA SI } & \multicolumn{2}{|c|}{ BACTERIEMIA NO } \\
\hline & $\begin{array}{c}\text { Todos } \\
\text { los pacientes }\end{array}$ & HOSPITALIZADO & $\begin{array}{c}\text { CENTRO } \\
\text { PERIFÉRICO }\end{array}$ & HOSPITALIZADO & $\begin{array}{c}\text { CENTRO } \\
\text { PERIFÉRICO }\end{array}$ \\
\hline $\mathrm{N}^{0}$ Pacientes & 173 & $22\left(10^{*}\right)$ & $38\left(10^{*}\right)$ & 81 & 43 \\
\hline Media de Edad & $\begin{array}{l}68 \pm 13 \\
(31-93)\end{array}$ & $\begin{array}{l}71 \pm 13 \\
(31-91)\end{array}$ & $\begin{array}{l}72 \pm 14 \\
(31-91)\end{array}$ & $\begin{array}{l}71 \pm 13 \\
(36-92)\end{array}$ & $\begin{array}{l}69 \pm 12 \\
(39-93)\end{array}$ \\
\hline Sexo (Mujeres) & $64(37 \%)$ & $6(27 \%)$ & $13(34 \%)$ & $48(38 \%)$ & $16(37 \%)$ \\
\hline Diabetes Mellitus & $80(46 \%)$ & $20(90 \%)$ & $22(57 \%)$ & $32(39 \%)$ & $16(37 \%)$ \\
\hline Media tiempo con CVC & $\begin{array}{c}526 \pm 445 \\
(1-1.468)\end{array}$ & $\begin{array}{l}899 \pm 480 \\
(39-1.468)\end{array}$ & $\begin{array}{l}823 \pm 462 \\
(75-1.468)\end{array}$ & $\begin{array}{l}506 \pm 414 \\
(30-1.467)\end{array}$ & $\begin{array}{l}260 \pm 290 \\
(1-1.352)\end{array}$ \\
\hline Media $n^{\circ}$ CVC/paciente & $\begin{array}{l}2,09 \pm 1,74 \\
\quad(1-11)\end{array}$ & $\begin{array}{c}4 \pm 2,6 \\
(1-11)\end{array}$ & $\begin{array}{c}3,4 \pm 2,4 \\
(1-11)\end{array}$ & $\begin{array}{c}2 \pm 1,4 \\
(1-7)\end{array}$ & $\begin{array}{c}1 \pm 0,5 \\
(1-3)\end{array}$ \\
\hline Media días hospitalizado & $\begin{array}{l}23 \pm 41 \\
(0-721)\end{array}$ & $\begin{array}{c}68,6 \pm 71 \\
(2-251)\end{array}$ & $\begin{array}{l}54 \pm 65 \\
(0-1.453)\end{array}$ & $\begin{array}{l}19 \pm 30 \\
(0-181)\end{array}$ & 0 \\
\hline Media BRC/paciente & $0,6 \pm 1,2(0-8)$ & $2,5 \pm 1,9(1-8)$ & $2,4 \pm 1,6(1-6)$ & 0 & 0 \\
\hline
\end{tabular}

* 10 pacientes padecieron BRC en ambos escenarios. 
El $90 \%$ de los pacientes que tuvieron algún episodio de BRC estando ingresado eran diabéticos, mientras que en el grupo de pacientes ingresados que no tuvieron ninguna BRC lo eran el $38 \%\left(x^{2}, p<0,001\right)$.

La media de tiempo con CVC hospitalizado fue de 899 días con BRC frente a 506 días sin BRC $(p<0,001)$. En el CP con BRC fue de 823 días y sin BRC 260 días $(p<0,001)$.

La media de CVC por paciente hospitalizado fue de 4 CVC cuando hubo BRC y 2 CVC sin BRC $(p<0,05)$. En el CP 3,4 CVC con BRC y 1 CVC $\sin B R C(p<0,05)$.

De los 172 pacientes, 48 sufrieron algún episodio de BRC. En la Figura 1 se describe el número de BRC por paciente.

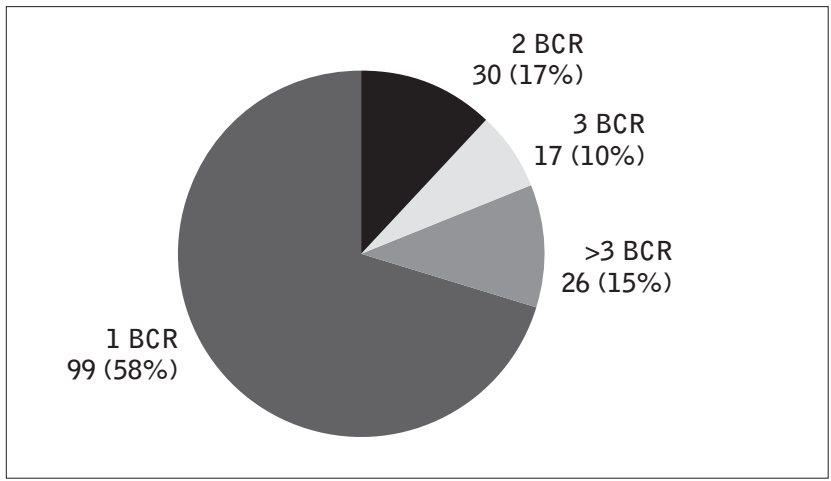

Figura 1. Número de episodios de BRC/paciente.

\section{CVC:}

Durante el período de estudio el $48 \%$ de los pacientes tuvo 1 solo CVC, el $20 \% 2$ CVC, el $12 \% 3$ CVC y el $19 \%$ más de 3 CVC.

El número de hospitalizaciones fue de 648 . El $28 \%$ de los pacientes incluidos no tuvo ningún ingreso hospitalario.

\section{Hemocultivos:}

Se realizaron un total de 475 hemocultivos; el $78 \%$ fueron negativos. Se obtuvieron 102 cultivos positivos: 71 en pacientes no hospitalizados y 31 en pacientes hospitalizados. En la Tabla 2 se presentan los gérmenes aislados en los hemocultivos positivos en el CP y durante la hospitalización.
Tabla 2. Gérmenes aislados en los hemocultivos positivos.

\begin{tabular}{l|c|c|}
\hline & $\begin{array}{c}\text { PACIENTES } \\
\text { HOSPITALIZADOS }\end{array}$ & $\begin{array}{c}\text { PACIENTES } \\
\text { CENTRO } \\
\text { PERIFERIC0 }\end{array}$ \\
\hline Staphylococcus epidermidis & $51,6 \%$ & $16,7 \%$ \\
Staphylococcus hominis & $16,1 \%$ & $11,1 \%$ \\
\hline Staphylococcus aureus & $12,9 \%$ & $20,8 \%$ \\
\hline Staphylococcus aureus MRSA & $6,5 \%$ & $2,8 \%$ \\
\hline Enterococcus faecalis & $3,2 \%$ & $2,8 \%$ \\
\hline Enterobacter cloacae complex & $3,2 \%$ & $1,4 \%$ \\
\hline Staphylococcus haemolyticus & $3,2 \%$ & $1,4 \%$ \\
Staphylococcus warneri & $3,2 \%$ & $1,4 \%$ \\
\hline Escherichia coli & & $6,9 \%$ \\
Staphylococcus capitis & & $5,6 \%$ \\
\hline Kleibsella & & $4,2 \%$ \\
\hline Pseudomonas aeruginosa & & $4,2 \%$ \\
\hline Otros & & $21 \%$ \\
\hline
\end{tabular}

En la Tabla 3 se muestran las tasas de BRC en dos períodos de tiempo: 2014-2015 vs 2016-2017, y en dos escenarios: Hospital donde siempre se selló con heparina $5 \%$, y $\mathrm{CP}$ en el que durante el primer período se selló el CVC con heparina 5\%, en el segundo período con Citra-Lock ${ }^{\mathrm{TM}}$.

\section{Tasas de BRC/hospitalización:}

En la Tabla 4 se representan las BRC en pacientes hospitalizados vs CP. La tasa de BRC en pacientes hospitalizados fue de 7,81 BRC/1000 días/CVC, y en los pacientes no hospitalizados de 0,81 BRC/1000 días/CVC.

Tabla 3. Tasas de BRC sellado: 2014 y 2015 vs 2016 y 2017.

\begin{tabular}{|c|c|c|c|}
\hline & $2014-2015$ & 2016-2017 & \\
\hline & \multicolumn{2}{|c|}{ HOSPITALIZADO } & \\
\hline Sellado & Heparina 5\% & Heparina 5\% & \\
\hline Días & 1.945 & 2.117 & OR 0,67 \\
\hline BRC & 19 & 14 & (IC:0,74-2,95) \\
\hline \multirow[t]{2}{*}{ BRC $\times 1000$ días CVC } & 9,77 & 6,61 & $p=0,27$ \\
\hline & \multicolumn{2}{|c|}{ CP } & \\
\hline Sellado & Heparina 5\% & Citra-Lock ${ }^{\mathrm{TM}}$ & \\
\hline Días & 37.998 & 49.160 & \multirow{3}{*}{$\begin{array}{c}\text { OR } 0,77 \\
\text { (IC: } 0,48-1,23) \\
p=0,28\end{array}$} \\
\hline BRC & 35 & 35 & \\
\hline BRC x 1000 días CVC & 0,92 & 0,71 & \\
\hline
\end{tabular}


Tabla 4. Tasas de BRC comparadas en pacientes hospitalizados y no hospitalizados.

\begin{tabular}{l|c|c|c}
\hline & Hospitalizado & CP & \\
\hline Edad & $76(71,2-80,8)$ & $68,3(64,2-72,2)$ & ns \\
\hline Sexo (Mujeres) & $33 \%$ & $40 \%$ & ns \\
\hline DM & $89 \%$ & $70 \%$ & $n s$ \\
Días CVC & 3.969 & 87.492 & \\
BRC & 31 & 71 & OR: 9,6 \\
BRC $\times 1000$ días CVC & 7,81 & 0,81 & IC $(6,3-14,6)$ \\
$\mathrm{p}=0,0001$
\end{tabular}

en 2007, y puso de manifiesto que aproximadamente el $50 \%$ de los enfermos con ERC inició el tratamiento mediante HD cada año a través de un CVC durante el período 2000-20117. En nuestro hospital en 2016 el $51 \%$ de los pacientes en HD que ingresaron eran portadores de $\mathrm{CVC}^{17}$. No hemos encontrado literatura que relacione directamente tasas de

La probabilidad (OR) de que se produzca una bacteriemia cuando el paciente es hospitalizado es 9,6 veces mayor que cuando el paciente no ingresa.

\section{Discusión}

Este estudio indica que los pacientes que se dializan en un $C P$ tienen significativamente más riesgo de padecer BRC relacionada con el CVC cuando son hospitalizados que cuando se dializan en su centro habitual. Si bien se observó una tendencia a reducirse la tasa de BRC cuando se empleó el Citra-Lock ${ }^{\mathrm{TM}}$ en el CP no hubo significación estadística, por lo que estos resultados son independientes del sellado utilizado.

El estudio de Beathard y Urbanes ${ }^{18}$, estudio referente en la evaluación de las tasas de BRC, las clasifica en función del número de episodios/ 1000 días CVC. Así, una tasa por debajo de un episodio/1000 días de CVC sería un resultado excelente pero más de 7 episodios/1000 días de CVC hablaría de resultados muy malos. Basándonos en estos datos, nuestros resultados en el CP (0,81 BCR/1000 días CVC) estarían en la excelencia. Por el contrario, la tasa de BRC en pacientes hospitalizados (7,81 BCR/1000 días CVC) indica un resultado negativo y preocupante. Este dato es más relevante si consideramos que en nuestro entorno el uso de CVC se mantiene por encima de los estándares recomendados por las guías de accesos vasculares ${ }^{1}$. De hecho, el grupo de estudio del Acceso Vascular español apunta el aumento del uso de CVC en pacientes prevalentes, en la comunidad de Madrid pasó de uno de cada 4 pacientes en 2007 a casi uno de cada 3 pacientes a final del año 20086. Otros estudios realizados en centros distribuidos por todo el territorio nacional, detectaron también este aumento en el uso del CVC, que pasó de un $7 \%$ en el estudio DOPPS 1 (2001) al $21 \%$ en el DOPPS $3(2007)^{8}$. Igualmente, el Registro de Enfermedades Renales de Cataluña mostró un aumento de pacientes dializados a través de un CVC del $12 \%$ en el 2002 frente al 19\%
BRC durante los ingresos y las que se producen en su centro habitual. El estudio de casos y controles de Fram et $\mathrm{a}^{19}$, con una población analizada de 162 pacientes en programa de HD señala la hospitalización previa como un factor de riesgo independiente de bacteriemia. En su grupo de estudio, la hospitalización previa aumentó la posibilidad de desarrollar infecciones del torrente sanguíneo en 6,6 veces. Los autores consideran que los pacientes que desarrollaron bacteriemia después del ingreso se podrían haber infectado durante su estancia hospitalaria. A pesar de que estos resultados son cercanos a nuestra hipótesis, debemos tomarlos con prudencia puesto que estudian todas las bacteriemias, incluidas aquellas en las que el foco primario no fue el CVC. Los mismos autores citan algunos estudios ${ }^{19-22}$ que relacionan la hospitalización previa con la infección por bacterias multirresistentes pero con la misma limitación en cuanto al origen de la bacteriemia.

El estudio de Sabatier et $\mathrm{a}^{23}$ refiere que entre un $5 \mathrm{y}$ un $10 \%$ de los pacientes que se hospitalizan van a padecer una infección nosocomial. Por su parte, el estudio de $D^{\prime}$ Agata et al $^{24}$ advierte que los pacientes de HD tienen un riesgo sustancialmente mayor de desarrollar una infección nosocomial en comparación con otros pacientes hospitalizados. Los hospitales terciarios y universitarios de tercer nivel tienen una incidencia mayor de $B R C^{4,24,25}$, que puede ser tres veces mayor que la de los centros no universitarios ${ }^{24}$. Otro estudio, de Ferrer et $\mathrm{al}^{4}$, relaciona el mayor riesgo de BRC con el empleo de catéteres transitorios, comorbilidad del enfermo, e ingreso en áreas de cuidados intensivos. El 15\% de los pacientes de nuestro estudio que fueron hospitalizados lo hizo en la UCI en algún momento del ingreso ${ }^{17}$. Este aspecto debe tenerse en cuenta, ya que el ingreso en UCI se asocia con mayor riesgo de bacteriemia nosocomial ${ }^{4,23}$, en algunos casos superior a 7,4 veces a la reportada en pacientes admitidos en otras áreas del hospital. Como cabía esperar, la elevada comorbilidad $^{3,4,23,26-29}$ es un factor de riesgo en todos los estudios. En nuestro caso, como en otros estudios $28,30-33$ ni el sexo, 
ni la edad, influyeron en la aparición de BRC, pero si la condición de ser diabético 23,27,28.

La suma de enfoques de estos diferentes estudios, que en general, concurren en nuestra población estudiada, puede ayudar a explicar la elevada tasa de BRC en los pacientes hospitalizados.

La comorbilidad de la población que precisa HD y el entorno hospitalario son factores no modificables. Sin embargo, sí lo es optimizar los cuidados de enfermería relacionados con los $\mathrm{CVC}^{1-5,9,11,18,25,26}$. La contaminación del punto de conexión de los CVC es la vía usual de colonización4, con el riesgo de progresar a través de la superficie intraluminal y formar la biocapa de colonización hasta llegar al extremo intravascular ${ }^{4,25}$. Es por ello que el uso del CVC fuera de las unidades de HD está lejos de las recomendaciones de las guías clínicas ${ }^{1}$. Sin embargo, en una población con problemas vasculares muy importantes ${ }^{17}$ su utilización es una realidad.

El tiempo con CVC es un factor de riesgo lógico de $\mathrm{BRC}^{1-3,11,12,14,16,21-23,27-29}$ tal como verificamos en nuestro estudio (Tabla 1) y muy relacionado con aspectos comórbidos que obligan a prolongar su empleo.

Los gérmenes aislados en el CP fue similar a los encontrados en otros estudios ${ }^{1,3,9,19,23,24,31-34}$ donde el estafilococo aureus fue el germen con mayor presencia. En cambio en el entorno hospitalario hay que sumar la importancia del estafilococo epidermidis, propio de estos catéteres de larga permanencia, considerado por mucho tiempo como un germen contaminante de cultivos pero que ahora se le reconoce como un patógeno importante en la infección de dispositivos endovenosos ${ }^{4}$.

La revisión canadiense de $B R C^{35}$ indica que un modelo de atención colaborativa que incluía un administrador de control de infecciones (una enfermera capacitada en las pautas actuales de manejo del catéter), disminuyó significativamente el número de BRC, y aunque se desarrollaba en un entorno extrahospitalario, extrapolarlo al ámbito hospitalario puede ser beneficioso. En este contexto el Servicio de Medicina Preventiva tiene un rol muy importante9,35.

El Grupo de Trabajo del Estudio Piloto: Bacteriemia Zero ${ }^{* 13}$ propone una intervención multifactorial que incluye varias actividades complementarias, en esta tiene gran peso la campaña "manos limpias" y la formación del personal sanitario en el manejo y prevención de complicaciones infecciosas relacionadas con CVC; así como medidas dirigidas a promover la cultura de seguridad en el trabajo diario. Dedicar especial atención a las medidas universales de asepsia 3 , 5, 13, 14,25 y en particular al lavado de manos es la medida preventiva más "barata".

\section{Limitaciones del estudio}

Es un estudio retrospectivo por lo que puede existir algún sesgo en la recogida de datos.

No se estudió la relevancia de la unidad asistencial, que podría aportar información complementaria de interés. Un porcentaje de los pacientes pudieron ser atendidos en un mismo ingreso en más de una unidad hospitalaria por lo que es difícil analizar su impacto.

A partir de los resultados obtenidos podemos concluir que la probabilidad (OR) de que se produzca una bacteriemia cuando el paciente es hospitalizado es 9,6 veces mayor que cuando el paciente no ingresa, y aunque esta observación no es generalizable (pudiendo ser en parte Centro dependiente) nos puede sugerir la necesidad de medir e interpretar las tasas de BRC en pacientes hospitalizados de manera independiente de los resultados globales.

Basándonos en estos resultados, además de las medidas generales conocidas, el objetivo prioritario debe ir dirigido a optimizar los protocolos de cuidados de CVC en unidades de enfermería, fuera de las salas de HD, menos familiarizadas con su uso.

Por último nos gustaría añadir que el rol de enfermería en el cuidado del CVC es protagonista para atenuar esta indeseable complicación.

\section{Conflicto de interés}

Los autores no presentan ningún conflicto de interés.

Recibido: 02-03-19

Revisado: 25-04-19

Modificado: 28-05-19

Aceptado: 5-06-19 


\section{Bibliografía}

1. Ibeas J, Roca-Tey R, Vallespín J, Moreno T, Moñux G, Martí-Monrós A, et al. Guía Clínica Española del Acceso Vascular para Hemodiálisis. Nefrología [Internet]. 2017;37(November):1-193. [Consultado 14 abril 2018]. Disponible en: http://linkinghub.elsevier.com/retrieve/pii/S0211699517302175.

2. Andreu $D$, Ángel M, Blanco H. Eventos infecciosos en pacientes en hemodiálisis. 2015;54-6.

3. Cobo PA, Clave $P$, Vascular-Catéter:-Bacteriemia-Acceso. Prevalencia de bacteriemias relacionadas con el catéter de hemodiálisis en una unidad hospitalaria. Enferm Nefrol Octubre-Diciembre [Internet]. 2013;16(4):229-34. [Consultado 14 abril 2018]. Disponible en: http://scielo.isciii.es/pdf/enefro/v16n4/original2.pdf.

4. Ferrer $C$, Almirante $B$. Infecciones relacionadas con el uso de los cateteres vasculares. Enferm Infecc Microbiol Clin. 2014;32(2):115-24.

5. Albalate $M$, García RP, de Sequera $P$, Alcázar $R$, Puerta M, Ortega $M$, et al. ¿Hemos olvidado lo más importante para prevenir las bacteriemias en pacientes portadores de catéteres para hemodiálisis?. Nefrologia. 2010;30(5):573-7.

6. Roca-Tey R,Arcos E, Comas J, Cao H, Tort J.Vascular access for incident hemodialysis patients in Catalonia: analysis of data from the Catalan Renal Registry (2000-2011). J Vasc Access. 2015;16(6):472-9.

7. Gruss E, Portoles J, Caro P, Merino JL, López-Sánchez P, Tato A, et al. Los modelos de atención al acceso vascular condicionan resultados heterogéneos en los centros de una misma comunidad. Nefrologia. 2010;30(3):310-6.

8. Ethier J, Mendelssohn DC, Elder SJ, Hasegawa T, Akizawa T, Akiba T, et al. Vascular access use and outcomes: an international perspective from the Dialysis Outcomes and Practice Patterns Study. Nephrol Dial Transplant. 2008 0ct;23(10):321926.

9. Zhang HH, Cortes-Penfield NW, Mandayam S, Niu $\mathrm{J}$, Atmar RL, Wu E, et al. Dialysis Catheter-related Bloodstream Infections in Patients Receiving Hemodialysis on an Emergency-only Basis: A Retrospective Cohort Analysis. Clin Infect Dis. 2019 Mar;68(6):1011-6.
10. Suzuki M, Satoh N, Nakamura M, Horita S, Seki G, Moriya K. Bacteremia in hemodialysis patients. World J Nephrol. 2016 Nov;5(6):489-96.

11. Soi V, Moore CL, Kumbar L, Yee J. Prevention of catheter-related bloodstream infections in patients on hemodialysis: challenges and management strategies. Int J Nephrol Renovasc Dis. 2016;9:95-103.

12. Nguyen DB, Lessa FC, Belflower $R, M u Y$, Wise $M$, Nadle J, et al. Invasive methicillin-resistant Staphylococcus aureus infections among patients on chronic dialysis in the United States, 2005-2011. Clin Infect Dis. 2013 Nov:57(10):1393-400.

13. Palomar Martínez M, Álvarez Lerma F, Riera Badía MA, León Gil C, López Pueyo MJ, Díaz Tobajas C, et al. Prevención de la bacteriemia relacionada con catéteres en UCI mediante una intervención multifactorial. Informe del estudio piloto. Med Intensiva. 2010;34(9):581-9.

14. Leonardi G, Finotto G, Talaia M, Nappo A, Dolla C, Di Vico MC, et al. ["Getting to zero infections" in hemodialysis]. G Ital Nefrol. 2015;32(5).

15. Collins AJ, Foley RN, Gilbertson DT, Chen S-C. United States Renal Data System public health surveillance of chronic kidney disease and end-stage renal disease. Kidney Int Suppl [Internet]. 2015;5(1):2-7. [Consultado 14 abril 2018]. Disponible en: http://linkinghub.elsevier.com/retrieve/pii/ S2157171615321006.

16. Katneni R, Hedayati SS. Central venous catheter-related bacteremia in chronic hemodialysis patients: Epidemiology and evidence-based management. Nat Clin Pract Nephrol. 2007;3(5):256-66.

17. Sánchez Villar I. Impacto de frecuencia de urgencias de una unidad extrahospitalaria. Enfermería Nefrológica. 2017;20(1):41.

18. Beathard GA, Urbanes A. Infection associated with tunneled hemodialysis catheters. Semin Dial. 2008;21(6):528-38.

19. Fram D, Okuno MFP, Taminato M, Ponzio V, Manfredi SR, Grothe C, et al. Risk factors for bloodstream infection in patients at a Brazilian hemodialysis center: a case-control study. BMC Infect Dis. 2015 Mar;15:158. 
20. Aktas E, Pazarli 0, Kulah C, Comert F, Kulah E, Sumbuloglu V. Determination of Staphylococcus aureus carriage in hemodialysis and peritoneal dialysis patients and evaluation of the clonal relationship between carriage and clinical isolates. Am J Infect Control. 2011 Jun;39(5):421-5.

21. Barbosa D, Lima L, Silbert S, Sader H, Cendoroglo $M$, Draibe $S$, et al. Evaluation of the prevalence and risk factors for colonization by vancomycin-resistant Enterococcus among patients on dialysis. Am J Kidney Dis. 2004 Aug;44(2):337-43.

22. Nguyen $D B$, Shugart $A$, Lines $C$, Shah $A B, E d-$ wards J, Pollock $D$, et al. National Healthcare Safety Network (NHSN) Dialysis Event Surveillance Report for 2014. Clin J Am Soc Nephrol. 2017 Jul;12(7):1139-46.

23. Sabatier C, Peredo R, Valles J. [Bacterial bloodstream infections in critical patients]. Med intensiva. 2009 0ct;33(7):336-45.

24. D'Agata EMC, Mount DB, Thayer V, Schaffner W. Hospital-acquired infections among chronic hemodialysis patients. Am J Kidney Dis [Internet]. 2000 Jun 1;35(6):1083-8. [Consultado 14 abril 2018]. Disponible en: https://doi.org/10.1016/S0272-6386 (00)70044-8.

25. Schweiger A, Trevino S, Marschall J. Nosocomial infections in dialysis access. Contrib Nephrol [Internet]. 2015;184:205-21. [Consultado el 14 abril 2018]. Disponible en: https://www.karger.com/Article/Pdf/ 366120.

26. Sociedad Española de Medicina Preventiva SP e H. Estudio Epine-Epps 2017. Estud EPiNE. 2017;28:1990-2017.

27. Sahli F, Feidjel R, Laalaoui R. Hemodialysis catheter-related infection: rates, risk factors and pathogens. J Infect Public Health. 2017 Jul;10(4):403-8.
28. Izoard S, Ayzac L, Meynier J, Seghezzi J-C, Jolibois $B$, Tolani ML. [Infections on catheters in hemodialysis: Temporal fluctuations of the infectious risk]. Nephrol Ther. 2017 Nov;13(6):463-9.

29. Coker MA, Black JR, Li Y, Varma R, Almehmi A, Abdel Aal $A K$, et al. An analysis of potential predictors of tunneled hemodialysis catheter infection or dysfunction. J Vasc Access. 2018 Nov. doi:1129729818809669.

30. Miller LM, Clark E, Dipchand C, Hiremath S, Kappel J, Kiaii M, et al. Hemodialysis Tunneled Catheter-Related Infections. Can J kidney Heal Dis. 2016;3:2054358116669129.

31. Carrasco Salinas C, Ruiz García M, Gómez López VE, Méndez Ordóñez R, López Andreu $M$, et al. Análisis de las hospitalizaciones por bacteriemia relacionada con el catéter de hemodiálisis. Enfermería Nefrológica. 2013;16(2):88-92. [Consultado 16 abril 2018]. Disponible en: https://dx.doi.org/10.4321/S225428842013000200004.

32. Menegueti MG, Betoni NC, Bellissimo-Rodrigues $F$, Romao EA. Central venous catheter-related infections in patients receiving short-term hemodialysis therapy: incidence, associated factors, and microbiological aspects. Rev Soc Bras Med Trop. 2017;50(6):783-7.

33. Miller LM, Clark E, Dipchand C, Hiremath S, Kappel $\mathrm{J}$, Kiaii $\mathrm{M}$, et al. Hemodialysis tunneled catheter-related infections. Can J Kidney Heal Dis. 2016;3(1).

34. Sejia V. Género Staphylococcus. Etiopatogenia microbiológica [Internet]. 2012;III(16):255-72. [Consultado 2 abril 2018]. Disponible en: http://www.higiene.edu.uy/cefa/2008/Staphylococcus.pdf.

35. Lata C, Girard L, Parkins M, James MT. Catheter-related bloodstream infection in end-stage kidney disease: a Canadian narrative review. Can J kidney Heal Dis. 2016;3:24.

Este artículo se distribuye bajo una Licencia Creative Commons Atribución-NoComercial 4.0 Internacional. https://creativecommons.org/licenses/by-nc/4.0/ 